\title{
A influência do gradiente sucessional e da frutificação de Merostachys aff. multiramea em uma comunidade de aves da Floresta com Araucária
}

\author{
Nicholas Kaminski' , Alessandro C. Angelo' \& Patricia A. Nicola²
}

\begin{abstract}
1. Universidade Federal do Paraná, Departamento de Ciências Florestais, Av. Pref. Lothário Meissner, 3400, Campus Jardim Botânico, 80210-170, Curitiba, PR, Brasil. (nicholas.kaminski@yahoo.com.br; alessandrocangelo@gmail.com)

2. Universidade Federal do Vale do São Francisco, Colegiado de Ciências Biológicas. Rod. BR 407, Km 12, Lote 543, s/n - C1, 56300-990. Petrolina, PE, Brasil. (patricia.nicola@univasf.edu.br)
\end{abstract}

Recebido 10 dezembro 2014

Aceito 13 abril 2016

DOI: $10.1590 / 1678-4766 e 2016002$

\begin{abstract}
The influence of the succession gradient and Merostachys aff. multiramea fruiting in a community of birds in the Araucaria Forest. The Mixed Rain Forest (Araucaria Forest) is currently restricted to less than 1\% of the original area, occurring in different succession stages and degrees of preservation. This paper presents the influence of the gradient of succession vegetation and the phenomenon of bamboo (Merostachys aff. multiramea) flowering and fruiting on a bird community in an area of Araucaria forest on Santa Catarina. Between 2009 and 2010, 20 monthly samples were performed using the methodology of capturing and marking using mist-nets in three successional stages (initial, intermediate and advanced). During sampling, the fruiting of bamboos began in January 2010, allowing an equivalent sampling before and after the event. A total of 557 individuals belonging to 77 species were captured. The highest relative abundances occurred to Haplospiza unicolor ( $\mathrm{AR}=20,80 \%$ ), due to the relationship of this species with bamboos in flowering. The highest relative abundance ocurred for $58,44 \%$ of the species, pattern already reported in studies using mist-nets in neotropical forests. The highest diversity was found in the initial stage $\left(H^{\prime}=3,45\right)$, followed by intermediate and advanced sucessional stages. However, the equitability is more pronounced in the intermediate stage. The Jaccard similarity index showed that the initial stage is the most different in their composition avifauna in relation with others. The results also showed that the opening of gaps due to the death of bamboos after flowering has a similar role that occurring with habitat fragmentation, with specific impacts in understory insectivores and understory omnivores.
\end{abstract}

KEYWORDS. Avifauna, Mixed Rain Forest, bamboos, forest succession.

RESUMO. A Floresta Ombrófila Mista (FOM) está restrita atualmente a menos de 1\% da área original, ocorrendo em diversos estágios sucessionais e graus de conservação. O presente trabalho apresenta a influência do gradiente de sucessão vegetacional e do fenômeno de floração e frutificação de Merostachys aff. multiramea em uma comunidade de aves em área de FOM em Santa Catarina. Entre os anos de 2009 e 2010 foram realizadas 20 amostragens mensais utilizando a metodologia de captura e marcação com o auxílio de redes-de-neblina em três estágios sucessionais (inicial, intermediário e avançado). Durante a amostragem, a frutificação das taquaras teve início em janeiro de 2010, permitindo uma amostragem equivalente antes e após o evento. Foram capturados um total de 557 indivíduos pertencentes a 77 espécies. As maiores abundâncias relativas ocorreram para Haplospiza unicolor $(\mathrm{AR}=20,80 \%)$, devido à relação desta espécie com os taquarais. As menores abundâncias ocorreram para 58,44\% dos táxons, padrão já reportado em estudos utilizando redes-de-neblina em ambientes florestais no neotrópico. A maior diversidade foi encontrada no estágio inicial $\left(\mathrm{H}^{\prime}=3,45\right)$, seguido pelo estágio intermediário e avançado. Porém, a equitabilidade foi mais pronunciada no estágio intermediário. O Índice de similaridade de Jaccard demonstrou que o estágio inicial é o mais diferenciado na composição de sua avifauna em relação aos demais. Os resultados também demonstraram que a abertura das clareiras devido à morte dos taquarais possui um papel similar ao ocorrente com a fragmentação de habitats, com impactos sobre grupos específicos, especialmente os insetívoros de estrato inferior e médio e onívoros de estrato inferior.

PALAVRAS-CHAVE. Avifauna, Floresta Ombrófila Mista, taquaras, sucessão.

A Floresta Ombrófila Mista, ou Floresta com Araucária, é uma fitofisionomia da Floresta Atlântica típica da região sul do Brasil que originalmente apresentava uma área de distribuição de aproximadamente $200.000 \mathrm{~km}^{2}$ (KLEIN, 1960; MAACK, 1981). Atualmente os remanescentes florestais deste ecossistema não perfazem mais do que $1 \%$ da área original e apresentam-se em diversos estágios sucessionais e graus de conservação (CASTELLA \& BRITEZ, 2004).

Nos estratos médios e sub-bosques mais evoluídos da Floresta Ombrófila Mista, as formas de agrupamentos de vegetação nas associações formadas com o pinheiro (Araucaria angustifolia (Bertol.) Kuntze) são distintas (MaAcK, 1981; Klein, 1984). Segundo Klein (1963), estes agrupamentos são formados principalmente por 
imbuia (Ocotea porosa (Ness \& Mart.) Barroso), taquaras (Merostachys spp.), cedro (Cedrela fissilis Vel.) e erva-mate (Ilex paraguariensis A.St.-Hil.), bem como por diversas espécies de mirtáceas.

Taquaras desempenham importante função na regeneração e, ecologicamente, representam papel fundamental na dinâmica do ecossistema florestal (SMITH et al. 1981; SANTos et al., 2012). Tal grupo tem como característica marcante a frutificação maciça e a eficiente sincronia entre os indivíduos da espécie (WIDMER, 1998; Liebsch \& Reginato, 2009). Pelo fato de serem vegetais semelparos, isto é, seu ciclo de vida termina com a morte dos indivíduos após a floração, o período vegetativo pode se estender por muitos anos e quando ocorre, promove modificações consideráveis na estrutura da floresta.

A floração e posterior frutificação das taquaras são responsáveis por eventos que envolvem o acréscimo de certas populações de animais como os roedores, ocasionando fenômenos conhecidos popularmente por "ratadas" (PEREIRA, 1941; GIOVANNONI et al., 1946; Olmos, 1996; JAKSIK \& Lima, 2003), assim como o aparecimento de elementos da avifauna, que embora muitas vezes ocorram localmente, acabam por se agrupar de maneira maciça em busca deste recurso (SICK, 1997; Sigrist, 2006; Cestari \& BerNardi, 2011; FrANZ et al., 2014).

Apesar da importância deste vegetal no agrupamento de algumas aves ser reconhecido e documentado (Olmos, 1996; Silveira, 1999; Reid et al., 2004; VASCONCElos et al., 2005; ARETA et al., 2009; CESTARI \& BERNARDI, 2011), o processo de modificação da paisagem sobre os demais elementos da comunidade de aves é pouco conhecido.

O presente trabalho apresenta a influência do gradiente de sucessão vegetacional e o impacto do fenômeno de floração e frutificação de Merostachys aff. multiramea Hackel. sob uma comunidade de aves em área de Floresta Ombrófila Mista em Santa Catarina.

\section{MATERIAL E MÉTODOS}

Área de estudo. A Fazenda Santa Alice (FSA) localizase no município de Rio Negrinho, região do planalto norte

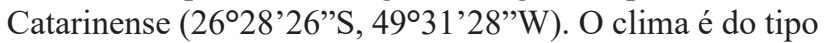
subtropical úmido mesotérmico $(\mathrm{Cfb})$ com ocorrência de geadas severas e verões frescos e as precipitações médias anuais são de $1.600 \mathrm{~mm}$ (ÁlLVARES et al., 2014).

A região onde a FSA está inserida é caracterizada pela produção de Pinus taeda L. em grande escala. Entremeado ao longo dos talhões de $P$. taeda, em maciços florestais ou ao longo do curso dos rios, ocorre um mosaico de ambientes naturais conectados, onde a vegetação da Floresta Ombrófila Mista se expressa em ambientes florestais distintos que são reconhecidos de acordo com seu estágio sucessional:

(1) Estágio inicial. A área amostrada possui cerca de 50 ha, compreendendo formações que não ultrapassam os 4 metros de altura, caracterizados pela dominância de Baccharis dracunculifolia DC. Em menor quantidade também registra-se Schinus terebinthifolia Raddi, Piptocarpha angustifolia Dusén ex Malme, Vernonanthura discolor (Spreng.) H.Rob., Myrsine coriacea (Sw.) R.Br. ex Roem. $\&$ Schult., Solanum mauritianum Scop., Miconia cinerascens Miq., Rhamnus sphaerosperma $\mathrm{Sw}$. e uma grande quantidade de plântulas de diversas espécies, dentre as mais significativas Ilex paraguariensis, Zanthoxylum rhoifolium Lam., Ocotea puberula (Rich.) Nees, Ocotea pulchella (Nees e Mart.) Mez e Vitex megapotamica (Spreng.) Moldenke. Neste local as taquaras ocorrem em densidade baixa, geralmente nas bordas próximas a estágios sucessionais mais avançados.

(2) Estágio intermediário. Área amostrada com cerca de 30 ha, caracterizada pela bracatinga (Mimosa scabrella Benth.) distribuída esparsamente, com árvores senescentes e um dossel com cerca de 8-10 m de altura. Destacam-se além da bracatinga, Vernonanthura discolor e Piptocarpha angustifolia. Aproximando-se de um estágio secundário mais desenvolvido, estes agrupamentos são substituídos gradativamente por Matayba elaeagnoides Radlk., Prunus brasiliensis (Cham. \& Schltdl.) D.Dietr., Drimys brasiliensis Miers, Nectandra megapotamica (Spreng.) Mez e Ilex paraguariensis. Há ainda a ocorrência marcante de Miconia petropolitana Cogn., M. cinerascens, Rhamnus sphaerosperma e plântulas de Cedrela fissilis, Ocotea puberula e Ocotea pulchella compondo o estrato logo abaixo dos grupos supracitados. Os taquarais são observados em pequenos agrupamentos, em densidade superior ao encontrado no estágio inicial, entretanto não dominam o sub-bosque local.

(3) Estágio avançado. Remanescente de aproximadamente $70 \mathrm{ha}$, caracterizado por uma floresta com a sinúsia superior alcançando $15-20 \mathrm{~m}$ de altura, representado pela ocorrência de Lauraceae, especialmente Ocotea puberula, O. pulchella, Nectandra megapotamica e em menor quantidade, Ocotea odorifera (Vell.) Rowher em destaque, com Sloanea lasiocoma K. Schum. e Cedrela fissilis. No estrato abaixo é encontrada com frequência Nectandra lanceolata Nees \& Mart., Casearia decandra Jacq., Vitex megapotamica e Ilex paraguariensis. O subbosque é formado por Psychotria suterella Müll.Arg., Leandra laevigata Cogn. e Myrcia splendens (Sw.)DC. Grandes adensamentos de taquara ocorrem no sub-bosque, dominando extensões consideráveis da área amostrada.

Esforço amostral. Entre 2009 e 2010, foram realizadas 20 amostragens mensais, ou seja, 10 em cada ano, utilizando a metodologia de captura e marcação, com o auxílio de redesde-neblina. Em cada um dos estágios sucessionais (inicial, intermediário e avançado), foi aberta uma transecção onde foram instaladas duas redes ( $15 \times 2,5$ - malha $25 \mathrm{~mm}$ ), que eram abertas nas primeiras horas da manhã e fechadas ao anoitecer, sendo revisadas a cada 30 minutos. Todos os indivíduos capturados foram marcados com anilhas metálicas disponibilizadas pelo ICMBio/CEMAVE. O enquadramento taxonômico seguiu o proposto por CBRO (2014).

Em janeiro de 2010 teve início a frutificação de Merostachys aff. multiramea, a qual ocasionou modificação na estrutura dos ambientes, através da abertura de clareiras em áreas anteriormente sombreadas ou com adensamento 
de vegetação, modificando consideravelmente a paisagem. A fim de se verificar mudanças na comunidade de aves antes e após a frutificação, as espécies capturadas foram categorizadas em guildas (grupos ecológicos funcionais), considerando a natureza do item alimentar, estrato e substrato de forrageamento e período de atividade, seguindo o proposto por WiLlis (1979): Insetívoros de solo (Is); Insetívoros de estrato inferior (Ieb); Insetívoros de estrato médio (Iem); Insetívoros de copa (Ic); Insetívoros de bambuzais e brenhas (Ibb); Insetívoros de tronco e galho (Itg); Granívoros de borda $(\mathrm{Gb})$; Onívoros de estrato inferior (Oei); Onívoros de copa (Oc); Onívoros e frugívoros de borda e interior (Ofbi); Frugívoros e onívoros de copa (Foc) e Nectarívoros (Ne).

Análises estatísticas. A curva de acumulação de espécies e a estimativa de riqueza com base no uso do estimador Jacknife foi calculada a partir de 500 adições aleatórias das amostras, utilizando o programa EstimateS 8.0 (Colwell, 2004).

A abundância relativa (AR) foi calculada considerando apenas os indivíduos capturados nas redes-de-neblina através da fórmula: $\mathrm{AR}=\mathrm{n}(100 / \mathrm{N})$, onde $\mathrm{n}$ é o número de indivíduos capturados de cada espécie e $\mathrm{N}$ é o total de indivíduos capturados em todo período amostral (MAGURRAN, 2011). De acordo com sua abundância, as espécies foram divididas em três categorias: (a) baixa, de um a três indivíduos capturados; (b) média, de quatro a 10 indivíduos e (c) alta, acima de 11 indivíduos capturados. Esta divisão foi realizada considerando a média do número de indivíduos capturados, excluindo-se Haplospiza unicolor Cabanis, 1851, a qual apresentou um $\mathrm{n}$ amostral muito superior as demais.

Foram comparadas diferenças na composição e abundância entre estágios distintos utilizando o Índice de Diversidade de Shannon-Wiener (H') (MAGURRAn, 2011) e a equitabilidade de Pielou (J') (Ludwig \& ReYnolds, 1988). O Índice H' foi comparado par a par, quanto a diferenças significativas, através do teste $t$ de Hutcheson (ZAR, 1999). A fim de se avaliar a similaridade entre a composição da avifauna nos diferentes estágios sucessionais, foi aplicado o Índice de Similaridade de Jaccard (IJ) (KREBS, 1999), com a confecção de um dendrograma entre as áreas.

Para se verificar possíveis diferenças na abundância das espécies antes e após o evento de frutificação das taquaras, foi realizado o teste t pareado (ZAR, 1999) para cada uma das guildas propostas, considerando o valor de $p<0,05$. Excluiu-se desta análise a guilda de insetívoros de borda e carnívoros, por estas contarem com apenas uma espécie.

\section{RESULTADOS}

Apesar de indicar uma leve estabilização, a curva cumulativa de espécies não atingiu a assíntota (Fig. 1). A estimativa de espécies aferida pelo Jackknife demonstrou que a riqueza deve ser superior (97,9 espécies), sendo necessário um esforço amostral mais prolongado para um real diagnóstico da comunidade de aves local.

Foram capturados 557 indivíduos pertencentes a 77 espécies (Tab. I). Este valor se refere ao somatório do



Fig. 1. Curva cumulativa de espécies de aves (linha sólida) e estimativa aferida pelo Jackknife (linha pontilhada) de amostragens realizadas em uma área de Floresta Ombrófila Mista, Rio Negrinho, SC, Brasil.

número de capturas $(\mathrm{n}=478)$ e recapturas $(\mathrm{n}=79)$. Durante o ano de 2009 foram capturados 280 indivíduos de 67 espécies, ao passo que em 2010 foram 198 capturas, referentes a 48 espécies. As famílias mais representativas foram Thraupidae $(n=12)$, Furnariidae $(n=8)$, Tyrannidae $(n=7)$, Dendrocolaptidae $(\mathrm{n}=6)$, Throchilidae, Rhynchocyclidae e Turdidae $(n=5)$.

As maiores abundâncias relativas ocorreram para Haplospiza unicolor Cabanis, 1851 ( $\mathrm{n}=99, \mathrm{AR}=20,80 \%$ ), Turdus albicollis Vieillot, 1818 e Myiothlypis leucoblephara (Vieillot, 1817) $(\mathrm{n}=24, \mathrm{AR}=5,04 \%)$, Tachyphonus coronatus (Vieillot, 1822) ( $\mathrm{n}=17, \mathrm{AR}=3,57 \%)$, Saltator similis d'Orbigny \& Lafresnaye, 1837 ( $\mathrm{n}=13, \mathrm{AR}=2,73 \%)$, Schiffornis virescens (Lafresnaye, 1838) e Lathrotriccus euleri (Cabanis, 1868) $(\mathrm{n}=12, \mathrm{AR}=2,52 \%)$, Conopophaga lineata Vieillot, $1818 \mathrm{e}$ Platyrinchus mystaceus (Wied, 1831) ( $\mathrm{n}=11, \mathrm{AR}=2,31 \%)$. Baixas abundâncias ocorreram para 45 espécies. Destas, 11 contaram com três capturas, 17 com duas capturas e outras $17 \mathrm{com}$ apenas uma captura. Outras 26 espécies obtiveram valores intermediários de abundância.

O estágio inicial obteve maior número de espécies $(\mathrm{n}=53)$, seguido pelo estágio avançado $(\mathrm{n}=41)$ e intermediário $(n=33)$. No entanto, o estágio avançado obteve maior número de capturas $(n=200)$ se comparado aos demais. A maior diversidade foi encontrada no estágio inicial $\left(H^{\prime}=3,45\right)$, seguido pelo estágio intermediário e avançado. Porém, a equitabilidade foi mais pronunciada no estágio intermediário (Tab. II).

$\mathrm{O}$ teste $\mathrm{t}$ de Hutcheson verificou diferenças significativas nos índices de diversidade do estágio inicial, quando comparado com os estágios intermediário $(\mathrm{t}=3,118$; $\mathrm{p}<0,05)$ e avançado ( $\mathrm{t}=3,620 ; \mathrm{p}<0,05)$. Não houve diferenças na comparação de H' entre estágio intermediário e avançado.

Do total de espécies, $29,87 \%$ foram exclusivamente capturadas no estágio inicial, $12,99 \%$ no estágio avançado e apenas 7,79\% foram exclusivas do estágio médio de sucessão.

O Índice de similaridade de Jaccard demonstrou que o estágio inicial é o mais diferenciado na composição de sua avifauna em relação aos demais (Fig. 2), especialmente se comparado ao estágio intermediário $(\mathrm{IJ}=0,63)$. A maior similaridade ocorreu entre os estágios médio e avançado 
Tab. I. Lista de espécies, guildas tróficas, locais de captura e abundâncias relativas das espécies amostradas durante os anos de 2009 e 2010 em uma área de Floresta Ombrófila Mista, Rio Negrinho, SC, Brasil (Ca, Carnívoros; Ibo, Insetívoros de Borda; Itg, Insetívoros de tronco e galho; Is, insetívoros de solo; Ibb, Insetívoros de bambuzais e brenhas; Iei, Insetívoros de estrato inferior; Iem, Insetívoros de estrato médio; Ic, Insetívoros de copa; Gb, Granívoros de borda; Oc, Onívoros de copa; Iei, Onívoros de estrato inferior; Ofbi, Onívoros e frugívoros de borda e interior; Foc, Frugívoros e onívoros de copa; Ne, Nectarívoros; A, captura no estágio Avançado; M, captura no estágio Intermediário; I, captura no estágio Inicial).

\begin{tabular}{|c|c|c|c|c|c|c|c|}
\hline Nome do Táxon & Nome popular & $\frac{\pi}{\Xi}$ &  & 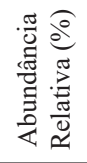 & 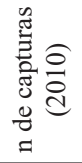 &  &  \\
\hline \multicolumn{8}{|l|}{ Accipitriformes } \\
\hline \multicolumn{8}{|l|}{ Accipitridae } \\
\hline Accipiter striatus Vieillot, 1808 & gavião-miúdo & $\mathrm{Ca}$ & 0 & - & 1 & 0,21 & M \\
\hline \multicolumn{8}{|l|}{ Columbiformes } \\
\hline \multicolumn{8}{|l|}{ Columbidae } \\
\hline Leptotila rufaxilla (Richard \& Bernard, 1792) & juriti-gemedeira & Gf & 2 & 0,42 & 0 & - & A \\
\hline Geotrygon montana (Linnaeus, 1758) & pariri & Gf & 0 & - & 2 & 0,42 & A \\
\hline \multicolumn{8}{|l|}{ Apodiformes } \\
\hline \multicolumn{8}{|l|}{ Trochilidae } \\
\hline Phaethornis eurynome (Lesson, 1832) & rabo-branco-acanelado & $\mathrm{Ne}$ & 2 & 0,42 & 1 & 0,21 & A I \\
\hline Stephanoxis lalandi (Vieillot, 1818) & beija-flor-de-topete & $\mathrm{Ne}$ & 2 & 0,42 & 0 & - & M \\
\hline Thalurania glaucopis (Gmelin, 1788) & beija-flor-de-fronte-violeta & $\mathrm{Ne}$ & 3 & 0,63 & 0 & - & M I \\
\hline Leucochloris albicollis (Vieillot, 1818) & beija-flor-de-papo-branco & $\mathrm{Ne}$ & 4 & 0,84 & 1 & 0,21 & I \\
\hline Clytolaema rubricauda (Boddaert, 1783) & beija-flor-rubi & $\mathrm{Ne}$ & 1 & 0,21 & 1 & 0,21 & A I \\
\hline \multicolumn{8}{|l|}{ Trogoniformes } \\
\hline \multicolumn{8}{|l|}{ Trogonidae } \\
\hline Trogon rufus Gmelin, 1788 & surucuá-de-barriga-amarela & Ofbi & 6 & 1.261 & 0 & - & A M I \\
\hline \multicolumn{8}{|l|}{ Piciformes } \\
\hline \multicolumn{8}{|l|}{ Picidae } \\
\hline Picummus temminckii Lafresnaye, 1845 & pica-pau-anão-de-coleira & Itg & 3 & 0,63 & 1 & 0,21 & $\mathrm{I}$ \\
\hline Veniliornis spilogaster (Wagler, 1827) & picapauzinho-verde-carijó & Itg & 0 & - & 1 & 0,21 & $\mathrm{I}$ \\
\hline Dryocopus galeatus (Temminck, 1822) & pica-pau-de-cara-canela & Itg & 1 & 0,21 & 0 & - & M \\
\hline \multicolumn{8}{|l|}{ Passeriformes } \\
\hline \multicolumn{8}{|l|}{ Thamnophilidae } \\
\hline Dysithamnus mentalis (Temminck, 1823) & choquinha-lisa & Ieb & 1 & 0,21 & 2 & 0,42 & M I \\
\hline Thamnophilus caerulescens Vieillot, 1816 & choca-da-mata & Ieb & 4 & 0,84 & 0 & - & A I \\
\hline Mackenziaena leachii (Such, 1825) & borralhara-assobiadora & Ieb & 0 & - & 1 & 0,21 & $\mathrm{I}$ \\
\hline Drymophila rubricollis (Bertoni, 1901) & trovoada-de-bertoni & $\mathrm{Ibb}$ & 0 & - & 1 & 0,21 & M \\
\hline \multicolumn{8}{|l|}{ Conopophagidae } \\
\hline Conopophaga lineata (Wied, 1831) & chupa-dente & Is & 6 & 1.261 & 5 & 1,05 & A M I \\
\hline \multicolumn{8}{|l|}{ Grallariidae } \\
\hline Hylopezus nattereri (Pinto, 1937) & pinto-do-mato & Is & 2 & 0,42 & 0 & - & A \\
\hline \multicolumn{8}{|l|}{ Formicariidae } \\
\hline Chamaeza campanisona (Lichtenstein, 1823) & tovaca-campainha & Is & 2 & 0,42 & 1 & 0,21 & A M I \\
\hline Scleruridae & & & & & & & \\
\hline Sclerurus scansor (Ménétriès, 1835) & vira-folha & Is & 8 & 1.681 & 1 & 0,21 & A I \\
\hline Dendrocolaptidae & & & & & & & \\
\hline Sittasomus griseicapillus (Vieillot, 1818) & arapaçu-verde & Itg & 4 & 0,84 & 4 & 0,84 & A I \\
\hline Xiphorhynchus fuscus (Vieillot, 1818) & arapaçu-rajado & Itg & 7 & 1.471 & 0 & - & A M \\
\hline Campylorhamphus falcularius (Vieillot, 1822) & arapaçu-de-bico-torto & Itg & 1 & 0,21 & 0 & - & A \\
\hline Lepidocolaptes falcinellus (Cabanis \& Heine, 1859) & arapaçu-escamado-do-sul & Itg & 6 & 1.261 & 0 & - & A M \\
\hline Dendrocolaptes platyrostris Spix, 1825 & arapaçu-grande & Itg & 1 & 0,21 & 1 & 0,21 & A \\
\hline Xiphocolaptes albicollis (Vieillot, 1818) & arapaçu-de-garganta-branca & Itg & 3 & 0,63 & 0 & - & A M \\
\hline Furnariidae & & & & & & & \\
\hline Clibanornis dendrocolaptoides (Pelzeln, 1859) & cisqueiro & Ieb & 2 & 0,42 & 0 & - & I \\
\hline Anabacerthia amaurotis (Temminck, 1823) & limpa-folha-miúdo & Iem & 1 & 0,21 & 0 & - & A \\
\hline Philydor rufum (Vieillot, 1818) & limpa-folha-de-testa-baia & Iem & 5 & 1,05 & 0 & - & M I \\
\hline Heliobletus contaminatus Berlepsch, 1885 & trepadorzinho & Iem & 4 & 0,84 & 1 & 0,21 & A M \\
\hline Syndactyla rufosuperciliata (Lafresnaye, 1832) & trepador-quiete & Iem & 3 & 0,63 & 0 & - & A \\
\hline Cichlocolaptes leucophrus (Jardine \& Selby, 1830) & trepador-sobrancelha & Iem & 1 & 0,21 & 0 & - & A \\
\hline Synallaxis cinerascens Temminck, 1823 & pi-puí & $\mathrm{Ibb}$ & 2 & 0,42 & 0 & - & M I \\
\hline Synallaxis spixi Sclater, 1856 & joão-teneném & Ibb & 1 & 0,21 & 1 & 0,21 & $\mathrm{I}$ \\
\hline Pipridae & & & & & & & \\
\hline Chiroxiphia caudata (Shaw \& Nodder, 1793) & tangará & Oeb & 15 & 3.151 & 3 & 0,63 & A I \\
\hline Tityridae & & & & & & & \\
\hline Schiffornis virescens (Lafresnaye, 1838) & flautim & Oeb & 10 & 2.101 & 2 & 0,42 & A M \\
\hline Pachyramphus castaneus (Jardine \& Selby, 1827) & caneleiro & Ic & 0 & - & 1 & 0,21 & I \\
\hline Cotingidae & & & & & & & \\
\hline Procnias nudicollis (Vieillot, 1817) & araponga & Foc & 1 & 0,21 & 0 & - & $\mathrm{M}$ \\
\hline
\end{tabular}


Tab. I. Cont.

\begin{tabular}{|c|c|c|c|c|c|c|c|}
\hline Nome do Táxon & Nome popular & $\underset{0}{\frac{\pi}{3}}$ & 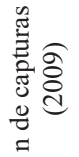 & 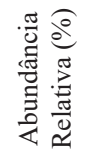 & 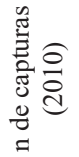 &  &  \\
\hline \multicolumn{8}{|l|}{ Cotingidae } \\
\hline Carpornis cucullata (Swainson, 1821) & corocochó & Foc & 3 & 0,63 & 0 & - & A M I \\
\hline \multicolumn{8}{|l|}{ Platyrinchidae } \\
\hline Platyrinchus mystaceus Vieillot, 1818 & patinho & Ieb & 9 & 1.891 & 2 & 0,42 & A M \\
\hline \multicolumn{8}{|l|}{ Rhynchocyclidae } \\
\hline Mionectes rufiventris Cabanis, 1846 & abre-asa-de-cabeça-cinza & Ic & 2 & 0,42 & 2 & 0,42 & A I \\
\hline Leptopogon amaurocephalus Tschudi, 1846 & cabeçudo & Ic & 1 & 0,21 & 1 & 0,21 & A M \\
\hline Phylloscartes ventralis (Temminck, 1824) & borboletinha-do-mato & Ic & 3 & 0,63 & 0 & - & I \\
\hline Tolmomyias sulphurescens (Spix, 1825) & bico-chato-de-orelha-preta & Iem & 2 & 0,42 & 0 & - & A \\
\hline Hemitriccus obsoletus (Miranda-Ribeiro, 1906) & catraca & $\mathrm{Ibb}$ & 2 & 0,42 & 0 & - & A \\
\hline \multicolumn{8}{|l|}{ Tyrannidae } \\
\hline Camptostoma obsoletum (Temminck, 1824) & risadinha & Ic & 1 & 0,21 & 0 & - & I \\
\hline Elaenia parvirostris Pelzeln, 1868 & guaracava-de-bico-curto & Ofbi & 3 & 0,63 & 0 & - & I \\
\hline Elaenia mesoleuca (Deppe, 1830) & tuque & Ofbi & 6 & 1.261 & 1 & 0,21 & I \\
\hline Phyllomyias virescens (Temminck, 1824) & piolhinho-verdoso & Iem & 1 & 0,21 & 1 & 0,21 & A I \\
\hline Attila phoenicurus Pelzeln, 1868 & capitão-castanho & Iem & 2 & 0,42 & 2 & 0,42 & M I \\
\hline Myiophobus fasciatus (Statius Muller, 1776) & filipe & Ibo & 2 & 0,42 & 1 & 0,21 & I \\
\hline Lathrotriccus euleri (Cabanis, 1868) & enferrujado & Ieb & 7 & 1.471 & 5 & 1,05 & A I \\
\hline \multicolumn{8}{|l|}{ Vireonidae } \\
\hline Cyclarhis gujanensis (Gmelin, 1789) & pitiguari & Ic & 5 & 1,05 & 2 & 0,42 & A M I \\
\hline Vireo chivi (Vieillott, 1817) & juruviara & Oc & 5 & 1,05 & 4 & 0,84 & M I \\
\hline Hylophilus poicilotis Temminck, 1822 & verdinho-coroado & Ic & 1 & 0,21 & 0 & - & M I \\
\hline \multicolumn{8}{|l|}{ Turdidae } \\
\hline Catharus fuscescens (Stephens, 1817) & sabiá-norte-americano & Ofbi & 1 & 0,21 & 0 & - & I \\
\hline Turdus flavipes Vieillot, 1818 & sabiá-una & Ofbi & 6 & 1.261 & 2 & 0,42 & A M \\
\hline Turdus rufiventris Vieillot, 1818 & sabiá-laranjeira & Ofbi & 13 & 2.731 & 6 & 1.261 & A M I \\
\hline Turdus amaurochalinus Cabanis, 1850 & sabiá-poca & Ofbi & 0 & - & 1 & 0,21 & I \\
\hline Turdus albicollis Vieillot, 1818 & sabiá-coleira & Oeb & 20 & 4.202 & 4 & 0,84 & A M I \\
\hline \multicolumn{8}{|l|}{ Passerellidae } \\
\hline Zonotrichia capensis (Statius Muller, 1776) & tico-tico & $\mathrm{Gb}$ & 3 & 0,63 & 2 & 0,42 & I \\
\hline \multicolumn{8}{|l|}{ Parulidae } \\
\hline Setophaga pitiayumi (Vieillot, 1817) & mariquita & Ic & 1 & 0,21 & 0 & - & I \\
\hline Basileuterus culicivorus (Deppe, 1830) & pula-pula & Ieb & 4 & 0,84 & 0 & - & A M I \\
\hline Myiothlypis leucoblephara (Vieillot, 1817) & pula-pula-assobiador & Is & 16 & 3.361 & 8 & 1.681 & A M I \\
\hline \multicolumn{8}{|l|}{ Thraupidae } \\
\hline Coereba flaveola (Linnaeus, 1758) & cambacica & $\mathrm{Ne}$ & 0 & - & 1 & 0,21 & I \\
\hline Saltator similis d'Orbigny \& Lafresnaye, 1837 & trinca-ferro-verdadeiro & Ofbi & 8 & 1.681 & 5 & 1,05 & A I \\
\hline Saltator maxillosus Cabanis, 1851 & bico-grosso & Ofbi & 1 & 0,21 & 1 & 0,21 & I \\
\hline Pyrrhocoma ruficeps (Strickland, 1844) & cabecinha-castanha & Ieb & 6 & 1.261 & 2 & 0,42 & A M I \\
\hline Tachyphonus coronatus (Vieillot, 1822) & tiê-preto & Ofbi & 8 & 1.681 & 9 & 1.891 & A I \\
\hline Lanio melanops (Vieillot, 1818) & tiê-de-topete & Oeb & 6 & 1.261 & 2 & 0,42 & A M I \\
\hline Stephanophorus diadematus (Temminck, 1823) & sanhaçu-frade & Oc & 2 & 0,42 & 1 & 0,21 & I \\
\hline Pipraeidea melanonota (Vieillot, 1819) & saíra-viúva & Oc & 1 & 0,21 & 1 & 0,21 & M I \\
\hline Haplospiza unicolor Cabanis, 1851 & cigarra-bambu & $\mathrm{Gb}$ & 7 & 1.471 & 92 & 19,33 & A M I \\
\hline Poospiza nigrorufa (d’Orb. \& Lafresnaye, 1837) & quem-te-vestiu & $\mathrm{Gb}$ & 0 & - & 2 & 0,42 & I \\
\hline Poospiza cabanisi Bonaparte, 1850 & tico-tico-da-taquara & $\mathrm{Gb}$ & 6 & 1.261 & 3 & 0,63 & I \\
\hline Sporophila caerulescens (Vieillot, 1823) & coleirinho & $\mathrm{Gb}$ & 1 & 0,21 & 0 & - & I \\
\hline \multicolumn{8}{|l|}{ Fringillidae } \\
\hline Sporagra magellanica (Vieillot, 1805) & pintassilgo & $\mathrm{Gb}$ & 0 & - & 2 & 0,42 & I \\
\hline
\end{tabular}

Tab.II. Resultados relativos à riqueza, abundância e diversidade de aves nos diferentes estágios sucessionais da Fazenda Santa Alice, Rio Negrinho, $\mathrm{SC}$, Brasil.

\begin{tabular}{lccc}
\hline & Inicial & Intermediário & Avançado \\
\hline Riqueza de espécies & 53 & 33 & 41 \\
Número de espécies capturadas & 192 & 86 & 200 \\
Numero de espécies exclusivas & $23(29,87 \%)$ & $6(7,79 \%)$ & $10(12,99 \%)$ \\
Índice de diversidade de Shannon-Wiener (H') & 3,45 & 3,08 & 3,03 \\
Equitabilidade (J') & 0,870 & 0,881 & 0,816 \\
\hline
\end{tabular}






Inicial

\begin{tabular}{cccccccc}
\hline & \multicolumn{1}{c}{} & $\mathbf{T}$ & $\mathbf{T}$ & $\mathbf{T}$ & $\mathbf{T}$ & \\
0.0 & 0.1 & 0.2 & 0.3 & 0.4 & 0.5 & 0.6 & 0.7
\end{tabular}

Distances

Fig. 2. Dendrograma de similaridade em três estágios sucessionais com os dados aferidos pelo Índice de Jaccard, em uma área de Floresta Ombrófila Mista, Rio Negrinho, SC, Brasil.

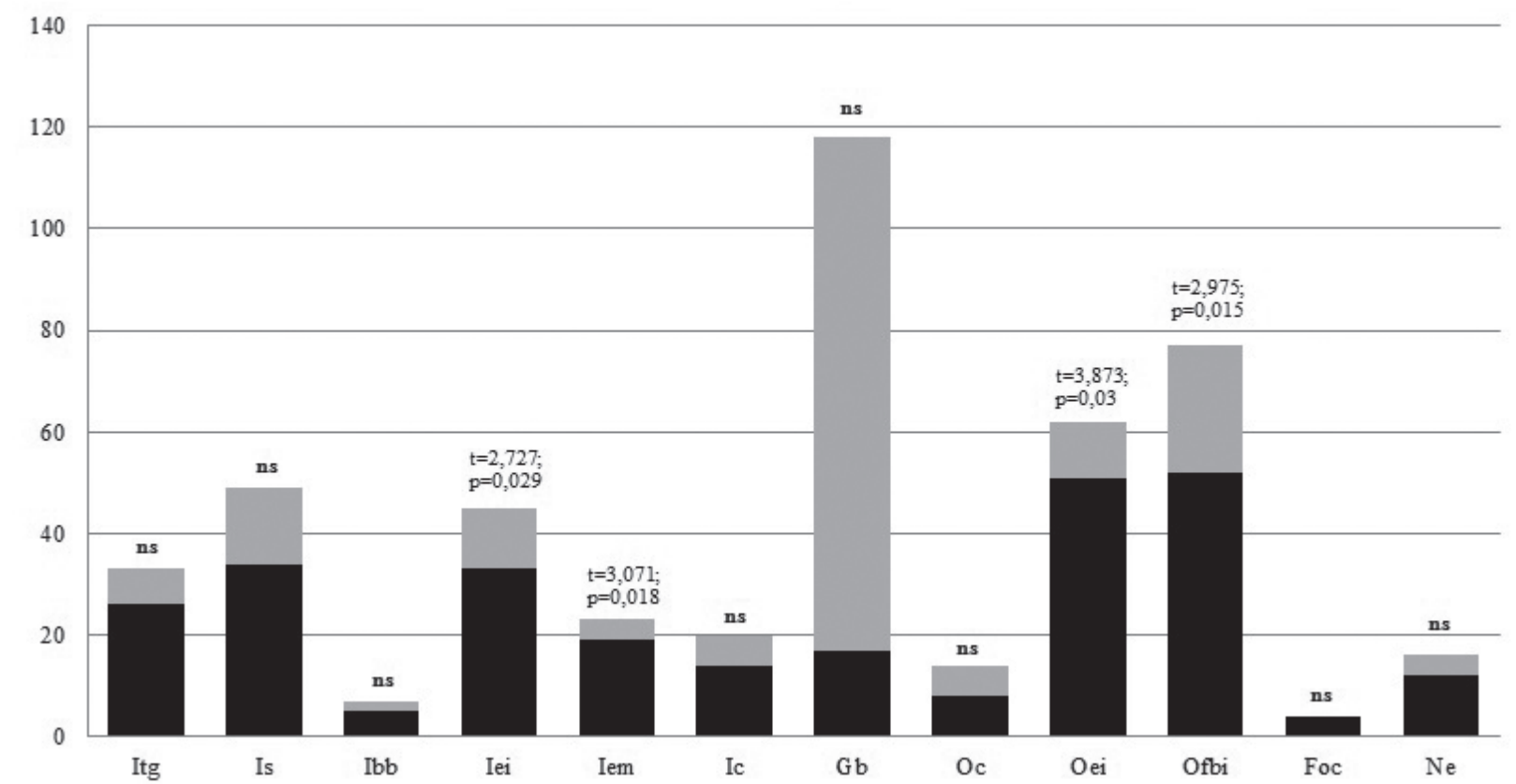

Fig. 3. Número de espécies de aves capturadas antes (preto) e após (cinza) o evento de frutificação da taquaras e os resultados do teste t para cada uma das guildas apresentadas, em uma área de Floresta Ombrófila Mista, Rio Negrinho, SC, Brasil (Itg, Insetívoros de tronco e galho; Is, Insetívoros de solo; Ibb, Insetívoros de bambuzais e brenhas; Iei, Insetívoros de estrato inferior; Iem, Insetívoros de estrato médio; Ic, Insetívoros de copa; Gb, Granívoros de borda; Oc, Onívoros de copa; Oei, Onívoros de estrato inferior; Ofbi, Onívoros e frugívoros de borda e interior; Foc, Frugívoros e onívoros de copa; $\mathrm{Ne}$, Nectarívoros).

( $\mathrm{IJ}=0,68)$, os quais possuem estruturas de vegetação similares.

Com relação à mudança da paisagem proporcionada pela seca dos taquarais sobre a avifauna verificou-se que os insetívoros de estrato médio $(\mathrm{t}=3,071 ; \mathrm{p}=0,018)$, onívoros e frugívoros de borda e interior $(\mathrm{t}=2,975 ; \mathrm{p}=0,015)$, insetívoros de estrato inferior $(\mathrm{t}=2,727 ; \mathrm{p}=0,029)$, e onívoros de estrato inferior $(t=3,873 ; p=0,03)$ foram as guildas tróficas que responderam as modificações a estrutura da floresta (Fig. 3).

\section{DISCUSSÃO}

Embora seja enfatizado o caráter de limitação do uso de redes de neblina para estudos ornitológicos (REMSEN \& GooD, 1996), trabalhos envolvendo a amostragem da avifauna de sub-bosque em florestas neotropicais são comuns
(Karr, 1977; BlaKe, 1989; Verea et al., 2000; Machado \& Fonseca, 2000; Verea \& Solórzano, 2001; MalletRodrigues \& Noronha, 2003; Scherer-Neto \& Toledo, 2012; Loures-RIBEIRo et al., 2011) e trazem resultados relevantes sobre a comunidade de aves. $\mathrm{O}$ uso de redes tem a vantagem de evitar registrar táxons de comportamento críptico e erros de observação (KARR, 1981; RAPPOLLE et al., 1998; SoDHI et al., 2004; BARLOW et al., 2006).

Devido à seletividade das redes-de-neblina na amostragem, a curva cumulativa de espécies não estabilizou, indicando a necessidade de maior esforço de captura para um diagnóstico mais preciso da comunidade de aves local. Mesmo na última amostragem, novas espécies ainda foram agregadas à lista final. Segundo MagurRan (2011), em ambientes tropicais exige-se um elevado esforço de campo 
para que ao menos 90\% das espécies sejam amostradas. Aliase ainda o fato de espécies pouco abundantes nas amostras influenciarem fortemente as curvas de acumulação, bem como as estimativas de riqueza.

Dentre as 77 espécies capturadas, 58,44\% tiveram baixa representatividade na amostra (até três indivíduos capturados). Este padrão, com a maioria dos táxons possuindo abundâncias baixas, reflete uma tendência nos estudos utilizando redes-de-neblina em florestas tropicais (KARR, 1977; KARR et al., 1990; BlAKE et al., 1990; VEREA \& Solórzano, 1998).

Apesar de MagurRan (2011) afirmar que estágios de sucessão avançados promovem maior heterogeneidade de habitats e consequente maior diversidade, nosso estudo verificou maior riqueza, diferenciação e número de espécies exclusivas no estágio inicial de sucessão. Este resultado pode ser explicado pelo fato do ambiente inicial apresentar formação vegetal com cerca de 4 metros de altura, fazendo com que a efetividade das redes se disponha a ser maior neste local. Segundo TERBORGH et al. (1990), a altura do dossel em florestas tropicais influencia diretamente nas taxas de captura.

Alguns grupos possuíram preferência pela ocupação de ambientes com estrutura florestal mais significativa. Dentre os seis representantes de Dendrocolaptidae, dois foram capturados apenas no estágio avançado [Campyloramphus falcularius (Vieillot, 1822) e Dendrocolaptes platyrostris Spix, 1825] e três no estágio avançado e intermediário [Xiphorynchus fuscus (Vieillot, 1818), Lepidocolaptes falcinellus (Cabanis \& Heine, 1859) e Xiphocolaptes albicollis (Vieillot, 1818)]. Esta correlação com a utilização de locais com uma estrutura de vegetação arbórea mais consistente vem a ser uma exigência ecológica de tal grupo (SICK, 1997). Por este motivo, alguns autores denotam a importância desta família como indicadora de qualidade dos ambientes florestais (AleIXo, 2001; AleiXo \& Vielliard, 1995; SoAres \& AnJos, 1999).

Destaca-se a presença de Cichlocolaptes leucophrus (Jardine \& Selby, 1830), Heliobletus contaminatus Berlepsch, 1885, Anabacerthia amaurotis (Temminck, 1823) e Syndactyla rufosuperciliata (Lafresnaye, 1832) apenas no estágio avançado de sucessão. Além da preferência por ambientes de caráter florestal e trechos de mata mais adensados (SicK, 1997), alguns estudos demonstram que estas espécies utilizam diversos microhabitats como bromélias, liquens, musgos e outras epífitas para forrageamento (PARRINI \& Pacheco, 2006; Cestari \& Pizo, 2008; Cestari, 2009; PARRINI et al., 2009). Tais exigências ecológicas estão em maior quantidade nas florestas bem estruturadas, caso da área em estágio avançado de sucessão.

Repartir a comunidade de aves em guildas torna-se uma importante ferramenta para se melhorar a precisão da análise dos dados obtidos, por agrupar espécies com adaptações ecológicas próximas (WILLIS, 1979; SOARES \& Anjos, 1999). A separação das espécies em grupos funcionais atribui um papel específico a cada categoria de espécies que desempenham uma atividade característica relacionada a processos biológicos do ecossistema, facilitando a interpretação de resultados. A análise por guildas tróficas revelou que houve variação na composição de quatro dos dez grupos analisados, em virtude de uma modificação da estrutura da floresta em decorrência da seca das taquaras.

Esperava-se um aumento dos granívoros na comunidade em virtude da frutificação das taquaras, porém o teste $\mathrm{t}$ não demonstrou valores significativos para este grupo. $\mathrm{O}$ único taxon que respondeu de maneira positiva foi Haplospiza unicolor, a qual costuma se agrupar suas populações em determinados locais, atraídas por taquarais em frutificação (Olmos, 1996; STOTz et al., 1996; Sick, 1997; VASCONCELlos et al., 2005; AreTA et al., 2009).

No ano de 2009, apenas sete indivíduos de $H$. unicolor haviam sido capturados, sendo um em área de estágio avançado de sucessão (local de maior adensamento das taquaras) e seis no estágio inicial. Em 2010, durante a frutificação de Merostachys sp., foram capturados 92 indivíduos, e apenas os meses de maio a julho foram responsáveis pela amostragem de 70 destes. Este período pode ser considerado como sendo o "pico" de frutificação, quando a maioria dos frutos estavam maduros. Se analisarmos a proporção de indivíduos capturados nestes três meses, $H$. unicolor representou $73,9 \%, 37,2 \%$ e $84,1 \%$ das capturas realizadas durante estas amostragens, respectivamente.

Taquarais são microhábitats particulares e sua dinâmica afeta a sobrevivência de aves e outros animais durante o período de frutificação (OLMOS, 1996; JAKSIC \& Lima, 2003; VAsConcelos et al., 2005; Areta et al., 2009). Pelo fato de tais agrupamentos fornecerem abrigo e presas em potencial para aves insetívoras (Olmos, 1996; SilveIRA, 1999; ReID et al., 2004; VASCONCELOS et al., 2005), estes grupos tendem a ser afetados com as clareiras formadas devido a senescência e morte das taquaras.

Os resultados demonstraram que a abertura das clareiras possui papel similar ao ocorrente com a fragmentação de habitats. A diminuição significativa das guildas de Insetívoros de estrato inferior $(t=2,727 ; p=0,0294)$ e Insetívoros de estrato médio $(t=3,071 ; p=0,018)$ refletem esta afirmação. Em estudos referentes ao impacto da fragmentação florestal na Mata Atlântica, os mesmos grupos tenderam a sofrer com a alteração do habitat (WILLIS, 1979; ALEIXo \& VIELLIARD, 1995; AleiXo, 1999; RibOn et al., 2003). CANADAY (1997) ao estudar o impacto da alteração no ambiente sobre as aves na Amazônia relatou que insetívoros apresentam maior sensibilidade a distúrbios em uma escala temporal curta, caso do evento em questão.

Com a diminuição da cobertura da vegetação, ambientes que anteriormente eram densos e sombreados passam a sofrer maior efeito de borda, o que acarreta maior incidência de luz solar e aumento de temperatura (Christiansen \& Pitter, 1997). Tal situação diminui a quantidade de habitats e microhabitats disponíveis e devem influenciar na disponibilidade de alimento, refletindo diretamente na riqueza e composição da comunidade de aves (Karr \& Roth, 1971; Simberloff \& Abelle, 1982). Reid et al. (2004), ao estudarem o papel dos bambus para as aves de sub-bosque, demonstraram a importância da densidade 
de taquaras para aves insetívoras, correlacionado a maior disponibilidade de alimento para esta guilda neste ambiente.

Aves que possuem preferência por locais de vegetação densa e sombreada (SICK, 1997; SIGRIST, 2006), como os onívoros de estrato inferior Chiroxiphia caudata (Shaw \& Nodder, 1793), Schiffornis virescens e Turdus albicollis também tenderam a diminuir a abundância após o período de frutificação das taquaras $(t=3,873 ; \mathrm{p}=0,03)$. As três espécies possuíam as maiores abundâncias relativas antes da frutificação das taquaras $($ T. albicollis $-\mathrm{n}=20$, $\mathrm{AR}=4,20 \% ; C$. caudata $-\mathrm{n}=15, \mathrm{AR}=3,15 \% ; S$. virescens $\mathrm{n}=10, \mathrm{AR}=2,10 \%$ ), sendo que após o evento suas capturas reduziram drasticamente. DíAz et al (2005) encontraram uma correlação positiva entre a riqueza e abundância de aves que ocupam o estrato inferior e sua ocorrência em florestas maduras com dossel emergente e sub-bosque dominado por taquaras, estrutura similar à existente no estágio avançado da FSA antes do evento de frutificação.

O resultado apresentado pelo teste t para os insetívoros de tronco e galho apresentou um valor muito próximo da significância $(t=2,2957 ; p=0,0508)$. $O$ valor constatado se deve principalmente à ausência de Lepidocolaptes falcinellus e Xiphorynchus fuscus após a abertura das clareiras propiciada pela seca dos taquarais. PARRINI \& PACHECO (2010) reportaram a semelhança destas duas espécies e sua utilização diferenciada de nichos em um mesmo ambiente. PoletTo et al. (2004), que caracterizaram o micro-habitat de cinco espécies de arapaçus no sul do Brasil, destacaram a preferência de $X$. fuscus por locais com sub-bosque denso. Tais preferências por habitats mais sombreados e densos pode exercer influência na utilização de áreas abertas por ambas as espécies e por este motivo, estas responderam significativamente a modificação da paisagem.

Em conclusão, nosso trabalho demonstrou que há diferenças na diversidade da avifauna de acordo com o estágio sucessional, denotando que áreas que possuem estágios sucessionais variados promovem maior diversidade na comunidade de aves.

Embora estágios avançados de sucessão possuam ambientes mais diversificados, as áreas em estágio inicial de sucessão promovem a ocorrência de uma parcela significativa das comunidade de aves dos ambientes analisados, possuindo grande importância na manutenção da avifauna local. Estas diferenças devem ser melhor investigadas através da adoção de metodologias complementares, haja visto a limitação das amostragens utilizando redes-de-neblina.

Foi verificado também que taquarais aumentam a heterogeneidade estrutural da floresta, promovendo maior diversidade de aves e sua senescência e morte após a frutificação altera a composição de alguns grupos funcionais, especialmente os relacionados ao estrato inferior e médio da floresta.

\section{REFERÊNCIAS BIBLIOGRÁFICAS}

Alerxo, A. 1999. Effects of selective logging on a bird community in the Brazilian Atlantic Forest. The Condor 101:537-548.
2001. Conservação da Avifauna da Floresta Atlântica: Efeitos da Fragmentação e a Importância de Florestas Secundárias In: Albuquerque, J. L. B.; CÂndido Jr, J. F.; Straube, F. C. \& Roos, A. L. Eds. Ornitologia e Conservação: Da Ciência às Estratégias. Tubarão, Unisul, p.199-206.

Aleixo, A. \& Vielliard, J. M. E. 1995. Composição e dinâmica da avifauna da mata de Santa Genebra, Campinas, São Paulo, Brasil. Revista Brasileira de Zoologia 12(3):493-511.

Álvares, C. A.; Stape, J. L.; Sentelhas, P. C.; Gonçalves, J. L. M. \& SparoveK, G. 2014. Köppen's climate classification map for Brazil. Meteorologische Zeitschrift 22(6):711-728.

Areta, J. I.; Bodrati, A. \& Coclke, K. 2009. Specialization on Guadua bamboo seeds by three bird species in the Atlantic forest of Argentina. Biotropica 41(1):66-73.

Barlow, J.; Peres, C. A.; Henriques, L. M. P.; Stouffer, P. C. \& WunderLe, J. M. 2006. The responses of understorey birds to forest fragmentation, logging and wildfires: An Amazonian synthesis. Biological Conservation 128:182-192.

BLAKE, J. G. 1989. Birds of primary forest undergrowth in western San Blas, Panama. Journal of Field Ornithology 60:178-189.

Blake, J. G.; Stiles, F. G. \& Loiselle, B. A. 1990. Birds of La Selva Biological Station: habitat use, trophic composition and migrants. In: Gentry, A. H. ed. Four Neotropical Rainforests. New York, Yale University Press, p.161-182.

Canaday, C. 1997. Loss of insectivorous birds along a gradient of human impact in Amazonia. Biological Conservation 77:63-77.

Castella, P. R. \& Britez, R. M. 2004. A floresta com araucária no Paraná: conservação e diagnóstico dos remanescentes florestais. Brasília, Ministério do Meio Ambiente. 233p.

CBRO - Comitê Brasileiro de Registros Ornitológicos. 2014. Listas das aves do Brasil - 11 ${ }^{\mathrm{a}}$ Edição. Disponível em $<\mathrm{http}$ ://www.cbro. org.br>. Acesso em 15.10.2014.

Cestari, C. 2009. Epiphyte plants use by birds in Brazil. Oecologia Brasiliensis 13(4):689-712.

Cestari, C. \& Bernardi, C. J. 2011. Predação do pixoxó Sporophila frontalis (Aves: Emberizidae) em sementes de Merostachys neesii (Poaceae: Babusoideae) durante um evento de frutificação em massa na floresta Atlântica. Biota Neotropica 11(3):407-411

Cestari, C. \& Pizo, M.A. 2008. Utilization of epiphytes by birds in a Brazilian Atlantic forest. Ornitologia Neotropical 19:97-107.

Christiansen, M. B. \& Pitter, E. 1997. Species loss in a forest bird community near Lagoa Santa in Southeastern Brazil. Conservation Biology 80:23-32.

Colwell, R. K. 2004. EstimateS 7.00: Statistical estimation of species richness and shared species from samples. Disponível em: $<\mathrm{http} / /$ viceroy.eeb.uconn.edu/estimates>.

Díaz, I. A.; Armesto, J. J.; Reid, S.; Sieving, K. E. \& Willson, M. F. 2005. Linking forest structure and composition: avian diversity in successional forests of Chiloe' Island, Chile. Biological Conservation 123:91-101.

Franz, I.; Barros, M. P. de; Cappelatti, L.; Dala-Corte, R. B. \& OtT, P. H. 2014. Birds of two protected areas in the southern range of the Brazilian Araucaria forest. Papéis Avulsos de Zoologia 54(10):111-127.

Giovannoni, M.; Vellozo, L. G. C. \& KubiaK, G. V. L. 1946. Sobre as "ratadas" do primeiro planalto paranaense. Arquivos de Biologia e Tecnologia 1:185-195.

JAKSIC, F. M. \& LIMA, M. 2003. Myths and facts about ratadas: bamboo blooms, rainfall peaks and rodent outbreaks in South America. Austral Ecology 28(3):237-251.

KARR, J. R. 1977. Ecological correlates of rarity in a tropical forest bird community. Auk 94:240-247.

1981. Surveying birds with mist nets. Studies in Avian Biology 6:62-67.

KARR, J. R. \& Roth, R. R. 1971. Vegetation structure and avian diversity in several new world areas. American Naturalist 105:423-435.

Karr, J. R.; Robinson, S. K.; Blake, J. G. \& BierregaArd, R. O. 1990. Birds of four Neotropical forests. In: Gentry, A. H. ed. Four Neotropical Rainforests. New York, Yale University Press, p. 237-269.

KLEIN, R. M. 1960. O aspecto dinâmico do pinheiro brasileiro. Sellowia 12:17-44.

1963. Observações e considerações sobre a vegetação do Planalto nordeste catarinense. Sellowia 15(15):39-56. 
1984. Aspectos dinâmicos da vegetação do Sul do Brasil. Sellowia 36:5-54.

Krebs, C. J. 1999. Ecological Methodology. 2ed. Menlo Park, AddisonWesley Educational Publishers. 619p.

Liebsch, D. \& Reginato, M. 2009. Florescimento e frutificação de Merostachys skvortzovii Sendulsky (taquara-lixa) no estado do Paraná. Iheringia, Série Botânica 64(1):53-56.

Loures-Ribeiro, A.; Manhães, M. A.; Dias, M. M.; Costa-Neto, S. J.; Silva, M. A. A.; Ribeiro, H. M. \& Lima, N. F. 2011. Aves de subbosque de uma área de Mata Atlântica de baixada do sudeste do Brasil. Ornithologia 4(2):76-85

Ludwig, J. A. \& Reynolds, J. F. 1988. Statistical Ecology: A primer on methods and computing. New York, John Wiley \& Sons, p. 89-94.

MAACK, R. 1981. Geografia física do Estado do Paraná. Rio de Janeiro, José Olympio Editora. 442p.

Machado, R. B. \& Fonseca, G. A. B. 2000. The avifauna of Rio Doce Valley, southeastern Brazil, a highly fragmented area. Biotropica 32:914-924.

Magurran, A. E. 2011. Medindo a diversidade biológica. Curitiba, Ed. UFPR. 261p.

Mallet-Rodrigues, F. \& Noronha, M. L. M. 2003. Variação na taxa de captura de passeriformes em um trecho de mata atlântica de encosta, no sudeste do Brasil. Ararajuba 11(1):111-118.

Olmos, F. 1996. Satiation or deception?: Mast-seeding Chusquea bamboos, birds and rats in the Atlantic forest. Revista Brasileira de Biologia 56(2):391-401

Parrini, R. \& Pacheco, J. F. 2006. Comportamento alimentar de Anabacerthia amaurotis (Passeriformes: Furnariidae) na Mata Atlântica Montana do Rio de Janeiro, Brasil. Atualidades Ornitológicas 132:4-7. . 2010. Comportamento de forrageamento de Xiphorhynchus fuscus (Passeriformes: Dendrocolaptidae) na Floresta Atlântica do Estado do Rio de Janeiro, sudeste do Brasil. Atualidades Ornitológicas 155:62-69.

PARrini, R.; PACHECO, J. F. \& RAJÃo, H. 2009. Comportamento alimentar de Heliobletus contaminatus (Passeriformes: Furnariidae) na Floresta Atlântica de altitude no sudeste do Brasil. Atualidades Ornitológicas 148:33-37.

Pereira, C. 1941. Sobre as "ratadas" no sul do Brasil e o ciclo vegetativo das taquaras. Arquivos do Instituto Biológico 12:175-195.

Polleto, F.; Anjos, L.; Lopes, E. V.; Volpato, G. H.; Serafini, P. P. \& FÁvaro, F. L. 2004. Caracterização do microhábitate vulnerabilidade de cinco espécies de arapaçus (Aves: Dendrocolaptidae) em um fragmento florestal do norte do estado do Paraná, sul do Brasil. Ararajuba 12:8996.

Rappole, J. H.; Winker, K. \& Powell, G. V. N. 1998, Migratory bird habitat use in southern Mexico: Mist nets versus point counts. Journal of Field Ornithology 69(4):635-643.

Reid, S.; Diaz, I. A.; Armesto, J. J. \& Willson, M. F. 2004. Importance of native bamboo for understory birds in Chilean temperate forests. Auk 121(2):515-525.

Remsen, J. V. \& Good, D. A. 1996. Misuse of data from mist-net captures to assess relative abundance in bird populations. Auk 113:381-398.
Ribon, R.; Simon, J. E. \& Mattos, G. T. 2003. Bird extinctions in Atlantic Forest fragments of the Viçosa Region, southeastern Brazil. Conservation Biology 17:1827-1839.

SAntos, S. C.; Budke, J. C. \& Muller, A. 2012 . Regeneração de espécies arbóreas sob a influência de Merostachys multiramea Hack. (Poaceae) em uma floresta subtropical. Acta Botanica Brasilica 26(1):218-229.

Scherer-Neto, P. \& Toledo, M. C. B. 2012. Bird community in an Araucaria forest fragment in relation to changes in the surrounding landscape in Southern Brazil. Iheringia, Série Zoologia 102(4):412-422.

SICK, H. 1997. Ornitologia brasileira. Rio de Janeiro, Nova Fronteira. 912p.

SIGRIST, T. 2006. Aves do Brasil: uma visão artística. São Paulo, Fosfetil. $672 \mathrm{p}$.

SilveIRA, M. 1999. Ecological aspects of bamboo-dominated forest in southwestern Amazonia: an ethnoscience perspective. Ecotropica 5:213-216

Simberloff, D. \& Abelle, L. G. 1982. Refuge design and island biogeographic theory: effects of fragmentation. American Naturalist 120:41-50

Smith, L. B.; Wasshausen, D. C. \& Klein, M. R. 1981. Gramíneas. Flora Ilustrada Catarinense, Parte I. Itajaí, Herbário Barbosa Rodrigues. $436 \mathrm{p}$.

SoARES, E. S. \& ANJos, L. 1999. Efeito da fragmentação florestal sobre aves escaladoras de tronco e galho na região de Londrina, norte do Paraná, Brasil. Ornitologia Neotropical 10(1):61-68

Sodhi, N. S.; Liow, L. H. \& Bazzaz, F. A. 2004. Avian extinctions from tropical and subtropical forests. Annual Review of Ecology, Evolution and Systematics 35:323-345.

Stotz, D. F.; Fitzpatrick, J. W.; Parker, T. A. \& Moskovits, D. K. 1996. Neotropical Birds: Ecology and Conservation. Chicago, University Chicago Press. 502p.

Terborgh, J.; Robinson, S. K.; Parker III, T. A.; Munn, C. A. \& Pierpont, N. 1990. Structure and organization of an Amazonian forest bird community. Ecological Monographs 60:213-238.

Vasconcelos, M. F.; Vasconcelos, A. P.; Viana, P. L.; Palú, L. \& Silva, J. F. 2005. Observações sobre aves granívoras (Colombidae e Emberezidae) associadas à frutificação de taquaras (Poaceae, Bambusoideae) na porção meridional de Cadeia do Espinhaço, Minas Gerais, Brasil. Lundiana 6(1):75-77.

Verea, C. \& SolórZANO, A. 1998. La avifauna del sotobosque de una selva decídua tropical en Venezuela. Ornitologia Neotropical 9:161-176.

.2001. La comunidad de aves del sotobosque de un bosque deciduo tropical em Venezuela. Ornitologia Neotropical 12:235-253.

Verea, C.; Fernández-Badillo, A. \& Solórzano, A. 2000. Variácion en la composición de las comunidades de aves de sotobosque de dos bosques en el norte de Venezuela. Ornitologia Neotropical 11:65-79.

WIDMER, Y. 1998. Flowering phenology of Chusquea bamboo with special to Chusquea talamancensis in Costa in Costa Rica. The Journal of the American Bamboo Society 1(2):1-20.

WILLIS, E. O. 1979. The compositon on avian communities in remanescent woodlots in southern Brazil. Papéis Avulsos de Zoologia 33(1):1-25.

ZAR, J. H. 1999. Biostatistical analysis. 4ed. Prentice Hall, New Jersey. 929p. 